Aus dem chemischen Laboratorium von Herrn Professor

Dr. Rosenfeld in Breslau.

\title{
Experimentelle und klinische Beiträge zur Frage der Hauttalgsekretion.
}

\author{
Von \\ Erich Kuznitzky, \\ Assistent an der Königlichen dermatologisehen \\ Universitätsklinik.
}

Das Studium der nach Bloch den Diathesen zuzurechnenden Hauterkrankungen, deren Gebiet vor nicht allzulanger Zeit noch völlig unerschlossen war, erfährt in den letzten Jahren, wenigstens in der deutschen Dermatologie, eine ständig wachsende Bereicherung. Namentlich die Befolgung der von Jadassohn postulierten funktionellen Prüfung hat im Tierexperiment sehr wichtige Aufschlüsse für das Verständnis gewisser Dermatosen geliefert - ich denke hier besonders an das bis dahin ganz dunkle Gebiet der Idiosynkrasien, auf welches die von Bruck zuerst hierfür erfolgreich verwandte Methode der passiven Übertragung der Anaphylaxie ein helles Licht warf. Dort jedoch, wo diesem Grundsatz Hindernisse erwachsen, müssen wir konstatieren, daß unsere Kenntnisse in den ersten Anfängen stecken geblieben sind. So reich unser Wissen von der Physiologie der verschiedenen Hautfunktionen ist, so wenig hat die Dermatologie - relativ - davon profitiert, eben weil es nicht möglich ist, durch das Experiment an einem warmblütigen Tier mit einer der menschlichen einigermaßen adäquaten Haut die vereinfachten Versuchsbedingungen, welche zum Studium der einzelnen Funktionen notwendig sind, zu schaffen. Hier sind besonders die Kenntnisse von der Sekretion der Haut und ihrer drüsigen Anhänge gemeint. Bei ihnen ist man lediglich auf das Experiment am Menschen angewiesen, 
wodurch sich gleichzeitig die Schwierigkeiten sehr erhöhen. Manchmal hilft hier ein glücklicher Zufall weiter, ich erinnere an die schönen Untersuchungen von Wechselmann und Loewy über die insensible Perspiration der Haut an drei blutsverwandten Personen mit Hemmungsbildungen des Hautdrüsensystems, bei denen durch die Atrophie der Talg- und Schweißdrüsen die erforderlichen vereinfachten Voraussetzungen für den Versuch gegeben waren. Solche glücklichen Zufälle sind aber äußerst selten, und wo sie nicht eintreffen, muß man eben mit den größten Hindernissen rechnen. Vor allem fehlt es am häufigsten an geeigneten Personen, welche nicht nur die Unbequemlichkeiten des Hautversuches, sondern oft auch noch die des Stoffwechselversuches willig ertragen und dabei zuverlässig beiben. Und selbst wenn solche Personen zur Verfügung stehen, können Versuche iäber den Umfang und die Größe der Hauttalgsekretion, denen wir uns hier namentlich zuwenden wollen, aus mancherlei nicht immer vorher zu übersehenden Gründen scheitern. So z. B. ist die Wahl der Versuchsperson hinsichtlich der Beschaffenheit ihres Unterhautfettgewebes nucht gleichgültig. Denn es will uns auf Grund mancher Versuche scheinen, daß fette Personen eine andere Talgausscheidung haben wie magere. Auch die Außentemperatur zurzeit des Versuches spielt sicher eine Rolle, die nicht außer acht gelassen werden darf, worauf wir uoch zurückzukommen haben werden.

Aber sicherlich der schwierigste Punkt bei der Durchfiihrung von Versuchen, welche die Menge des durch die Talgdrüsen abgeschiedenen Hautfettes eruieren sollen, liegt darin, $\mathrm{da} \beta$ es an einer geeigneten Methodik fehlte, bei welcher die Talgabscheidung der ganzen Haut oder wenigstens einer möglichst groß zu bemessenden Fläche derselben die Basis bei Berechnung der Resultate abgab.

Bisher behalf man sich auf folgende Weise:

Krukenberg, der erste, dem wir planmäßige Untersuchungen über die Talgsekretion der Haut zu verdanken haben, bediente sich eines, $2.5 \mathrm{qcm}$ großen Blättchens ungeleimten, festgefügten Papieres, welches der Haut des Sternums unmittelbar auflag. Das Blättchen blieb auf der Haut solange 
liegen, bis es sich, nach Bunsens photometrischem Verfahren untersucht, als mit Fett durchtränkt erwies."

Er kommt zu seinem Resultat, indem er weiterhin berichtet, daß unter Annahme der Körperoberfläche zu $16.000 \mathrm{qcm}$ und der Multiplikation mit 2400 „die von den Talgdrüsen täglich sezernierte Fettmenge demnach $40.8 \mathrm{~g}$ betragen würde".

Einige Zeit später wurden diese Untersuchungen von Leubuscher fortgesetzt, welcher ein 4 qom großes Stückchen Filtrierpapier auf die Haut auflegte und den Fettgehalt desselben nach verschieden langer Zeit dadurch bestimmte, daB er es mit dem Soxhlethschen Apparat extrahierte. Seine Resultate (zit. nach Rosenfeld) sind, dab das meiste Hautfett an der Stirn, weniger am Rücken, noch weniger an der Brust ausgeschieden würde; dann kommen in der Reihe nach abwärts Oberarm und Leib. Die Ausscheidung, beobachtet an fünf gleichartigen Versuchspersonen, wird auf $100 \mathrm{~g}$ pro Woche, d. h. also zirka $15 \mathrm{~g}$ pro Tag geschätzt. Nur bei Personen mit sehr starker Talgsekretion wurden auch die Krukenbergschen Zahlen erreicht. Schon ein oberflächlicher Vergleich der Ergebnisse dieser beiden Autoren zeigt deutlich, wie notwendig es bei derartigen Versuchen ist, eine möglichst große Hautfläche heranzuziehen, und zu welch großen Differenzen es führt, wenn man diese Forderung nicht berücksichtigt und nur einen so kleinen Teil der Körperoberfläche seinen Versuchen zu grunde legt, wie Krukenberg und Leubuscher es taten. Wir können daher Kosenfeld völlig beipflichten, wenn er die Methode der beiden vorgenannten Untersucher mit den Worten kritisiert:

"Man wird nicht verkennen, daß in den Versuchen beider Autoren die Fehlerquellen bei der Beobachtung enorm waren. Wenn man im Krukenbergschen Falle von $2.5 \mathrm{qcm}$ auf $16.000 \mathrm{qcm}$, welche Größe der Oberfläche des Menschen gegeben wurde, schloh, oder in den Experimenten von Leubuscher von $4 \mathrm{qcm}$ auf dieselben $16.000 \mathrm{qcm}$ schloß, die Beobachtungsziffer also mit 6400 oder mit 4000 multiplizieren mußte, so bätte die Methode der zu beobachtenden Fettausscheidung ein Wunder von Exaktheit sein müssen, wenn diese Resultate annähernd stimmen sollten. Statt dessen bediente 
sich Krukenberg der gänzlich unzuverlässigen photometrischen Methode. Genauer war ja immerhin die Bestimmung des Fettes nach Soxhleth, wie sie Leubuscher wählte. Aber auch sie enthält so viel Fehlerquellen (nur die Trocknung und Wägung zu nennen), daß sie eine Multiplikation mit 4000 unmöglich vertragen konnte, ohne zu den schreiendsten Unwahrscheinlichkeiten zu führen."

Hier setzen nun die Versuche Rosenfelds ein, welcher alle diese Fehler zu vermeiden trachtete und daher mehrere Änderungen in der Anordnung einführte. Zunächst ersetzte er das von Krukenberg und Leubuscher benutzte Papier durch eine Wolljacke und Wollhose, welche permanent Tag und Nacht getragen wurden und in welchen sich die Sekrete der Haut sammeln konnten. Es wurde also bierdurch der Hauttalg der gesamten Körperoberfläche mit Ausnahme des Kopfes, des Halses, der Hände und der Füße aufgefangen. Zweitens wurde eine exakte Extraktionsmethode eingeführt, indem nach Beendigung des Versuches die Wollwäsche in einem großen geschlossenen Glasgefäß mit Chloroform übergossen, mehrere Tage stehen gelassen und, nachdem das Chloroform durch ein Filter abgegossen war, mit einer eisernen Presse energisch ausgepreßt wurde. Nach Destillation des Chloroforms wurde der Rückstand in Äther gelöst, filtriert und getrocknet.

Schließlich wurde auch die Diät berücksichtigt, derart, daß während der Versuchszeit, welche bei den verschiedenen Personen 3,4, 5 bis 9 Tage dauerte, eine bestimmte Ernährungsweise eingeschlagen wurde. $\mathrm{Zu}$ letzterem Schritte führte die Absicht, die Kohlenhydrate und die Fette der Nahrung in ihrer eiweißsparenden Kraft zu vergleichen. Denn wenn die Kruk enbergschen und die Leubuscherschen Zahlen zu Recht bestanden, wenn täglich bis zirka $40 \mathrm{~g}$ Fett durch die Haut ausgeschieden wurden und dem Stoffwechsel verloren gingen, so mußte eine Minderwertigkeit der Fette schon aus dem Abgang von 370 Kalorien (im Hauttalg) vermutet werden. Deshalb suchte Rosenfeld sich mit dem eben geschilderten Verfahren einen Einblick in die wirkliche Größe des Verlustes zu verschaffen und ordnete seine Versuche derart an, daß er einer Periode, in welcher bei gleichbleibender EiweiB- und Kalorien- 
menge eine kohlenhydratreiche, aber fettarme Kost zugeführt wurde, eine solche von fettreicher, aber kohlenhydratarmer Nahrung folgen ließ. Hierdurch war natürlich die Möglichkeit gegeben, nicht nur den Umfang der Hauttalgausscheidung selbst, sondern auch einen etwaigen Einfluß der beiden Nährstoffe auf die Größe der Talgausscheidung zu studieren. Für die Kostanordnung möge ein Versuch, den Rosenfeld geschildert hat, als Beispiel dienen:

"Auf eine Kohlenhydratperiode, in welcher neben $250 \mathrm{~g}$ Fleisch, $20 \mathrm{~g}$ Kakao, $94 \mathrm{~g}$ Eier, an Fetten nur $70 \mathrm{~g}$ Butter, dagegen an kohlenhydrathaltiger Sustanz $100 \mathrm{~g}$ Zwieback und $332 \mathrm{~g}$ Zucker genossen wurden, folgte eine Fettperiode, in der die $332 \mathrm{~g}$ Zucker fortgelassen und durch die äquivalente Menge von $180 \mathrm{~g}$ Butter ersetzt wurde. Die Kost der zweiten Periode enthielt also statt $70 \mathrm{~g} 250 \mathrm{~g}$ Butter."

Die Ergebnisse, welche mit diesem Verfahren gewonnen wurden, hatten zunächst einen einheitlichen Charakter, indem erstens festgestellt wurde, daß die von der Haut während eines Tages ausgeschiedene Hauttalgmenge nur zirka 1-2 g betrug, also bedeutend geringer war, als man bisher annahm, und zweitens, daß eine Beeinflussung der Ernährung in der Hinsicht festgestellt werden konnte, daß in der Kohlenhydratperiode mehr Hautfett sezerniertwurde, als in der zweiten, der fettreichen, aber kohlenhydratarmen Versuchszeit.

Auf diesem Status setzten nun meine Versuche an mir selbst ein, welche zunächst eine Wiederholung und einfache Nachprüfung der bisherigen darstellten. Es wurden in einer viertägigen Periode $445 \mathrm{~g}$ Kohlenhydrate und zirka $50 \mathrm{~g}$ Fett genommen und dabei $2.057 \mathrm{~g}$ Hautfett produziert. In der sofort darauf folgenden Versuchszeit von drei Tagen wurden dagegen $200 \mathrm{~g}$ Fett und $100 \mathrm{~g}$ Kohlenhydrate zugefiuhrt und nur 1.7 $g$ Hauttalg pro die ausgeschieden. Während sich das Resultat dieses Versuches in nichts von den bisherigen unterschied, war hierbei aber schon insoweit eine Änderung getroffen, als die Vorbereitung der Wäsche einwandfreier gestaltet wurde. 
In den ersten Versuchen $\mathrm{R} o \mathrm{~s}^{\mathrm{e}} \mathrm{e}$ feld s war frischgekaufte oder gewaschene Wollwäsche benutzt worden, die in einigen Stichproben keinen wesentlichen Betrag an Chloroformextrakt ergeben hatte. Extrahierte man aber die zu verwendende Wollwäsche in dem sogleich zu schildernden längeren Verfahren, so ergaben sich doch hin und wieder Extraktzahlen von $1-5 g$, die nicht übersehen werden dürften. Es war natürlich, daß solche Fehlerquellen das Resultat bei einem Versuche von 3-4 Tagen eventuell beeinträchtigen konnten. Es wurde also die größte Sorgfalt auf eine möglichst genaue Extraktion der ungetragenen Wollwäsche verwendet in der Weise, daß diese vor dem Versuche so oft extrahiert wurde, bis das im Chloroformextrakt nachweisbare Fett auf die Menge von $0.002 g$ gesunken war. Dann wurde die Wäsche getrocknet und angezogen. Nach dem Versuche wurde die getragene Wäsche in der üblichen Weise extrahiert und die Extraktion so lange fortgesetzt, bis die niedrigste im Chloroformextrakt der ungetra. genen Wäsche nachgewiesene Fettzahl $(0.002 \mathrm{~g})$ wieder erreicht war. Ein Beispiel für die Handhabung dieses Verfahrens sei im folgenden angeführt:

Wollwäsche G., 5 Tage getragen, ergibt:

1. mal extrahiert, Chloroform abfiltriert . .2.902 $g$ Fett

2. $" n$ dto. . . 1.654 " "

3. $"$ dto. . $0.678 "$,

3. $" n$ Chloroform abgepreßt . $0 \cdot 161 "$ ",

4. $" \#$ abfiltriert ..0.185" "

4. " " " abgepreßt . $0.065 "$,

5. $" n$ abfiltriert . $0.007 "$,

$5 . " n$ abgepreßt . $0.002 "$,

Nunmehr suchten wir in neuen Versuchen Aufschlüsse darüber zu erhalten, ob Mastkost einen Einfluß auf die Hauttalgmenge habe und ob es dabei von Belang ist, mit welchen Stoffen, Kohlenhydraten oder Fetten, diese Mastzulage der Kost erreicht wird. Die Versuche stellte ich an mir, sowie an den Herren Kg., L. und G. an. Die Anordnung wurde so getroffen, daß außer der durch den Bedarf des Organismus bestimmten Kost eine Zulage von zirka $40 \%$ gereicht wurde, welche in der 
Kohlenhydratperiode aus etwa $200 \mathrm{~g}$ Rohrzucker, in der Fettperiode aus zirka $90 \mathrm{~g}$ Fett bestand. Das Fett wurde zwècks späteren Nachweises in Form von Sesamöl zugeführt, welches zu Remouladensauce verarbeitet genossen wurde. Bei den Versuchen, welche ich an mir anstellte, wurde der Kohlenhydratzeit eine kurze Vorperiode mit freier Kost ohne Zulage vorangeschickt.

Eine Übersicht über das Ergebnis der Mastversuche an mir selbst und an den anderen Versuchspersonen zeigt folgende Tabelle:

\begin{tabular}{|c|c|c|c|}
\hline & \multicolumn{3}{|c|}{ Mastrersuehe } \\
\hline & Vorperiode & $\begin{array}{c}\text { Kohlenhydr.- } \\
\text { Periode }\end{array}$ & Fettperiode \\
\hline Versuch K. I & 266 & $2 \cdot 12$ & $1 \cdot 91$ \\
\hline K. II & 0.99 & 068 & $0 \cdot 86$ \\
\hline Versuch L. & - & $0 \cdot 66$ & $1 \cdot 39$ \\
\hline$n \quad G$. & - & $1 \cdot 05$ & 0.98 \\
\hline Versuch Kg. . . & - & $1 \cdot 18$ & $1 \cdot 11$ \\
\hline
\end{tabular}

Das Resultat dieser Versuche war in mannigfacher Beziehung merkwürdig: 1. Während bei den früheren Versuchen die täglich ausgeschiedene Fettmenge in der Kohlenhydratperiode gewöhnlich $2 g$ oder nur wenig darunter betrug, lehrt ein Blick auf obige Tabelle, daß diese Zahl nur in einem einzigen von 5 Versuchen erreicht worden ist. Im Gegenteil, es hat den Anschein, als wenn in der Zeit der Masttage eine allgemeine Verringerung der Fettproduktion, ganz gleich, ob Kohlenhydrate oder Fettegereicht wurden, stattgefunden habe. Dafür spricht auch die Tatsache, daß, wenigstens in meinen beiden Versuchen, die Zahlen der Mastperiode hinter denen der Vorperiode, bei der ja die Zulage fehlte, nachstanden. 2. Ließ sich der bisher regelmäBigzu konstatierende Einfluß von Kohlenhydraten und Fetten auf die Talgsekretion bei den Mastrersuchen nicht wie sonst feststellen, vielmehr fand eine Verwischung, eine Nivellierung stellenweise sogar Umkehrung der Werte statt. 
Interessant war ferner die Feststellung, $d a B$ das in Form von Sesamöl gereichte Fett der Nahrung in den Hauttalg überging, wo es von uns mittels der Furfurolreaktion nachgewiesen werden konnte.

Bei einer Kritik der zunächst überraschenden obigen Resultate war nicht von der Hand zu weisen, daß gegenüber den in den ersten Versuchen gewonnenen Ergebnissen ins Gewicht fiel, daß hier die Wüsche, die während der Versuche getragen wurde, vorher sorgfältig extrahiert war und somit, da einige Gramm Fett aus ihr wegfallen konnten, eine niedrigere Zahl erreicht wurde. Da dies jedoch nicht immer der Fall war, mußte man annehmen, daß noch irgend ein anderer Faktor, der sich bisher unserer Kenntnis entzogen hatte, dahin wirkte, daß die Produktion des Hautfettes auf ein so niedriges Maß herabgedrückt wurde. Es fiel uns auf, daß während der meisten dieser Versuche, nicht aller, die Außentemperatur innerhalb der Versuchszeit verhältnismäßig niedrig war. Dies war besonders während der kalten Tage des Winters, des Herbstes und des Vorfrühlings der Fall. Es erhob sich also die Frage, ob dieser Umstand geeignet wäre, eine Brücke zu dem oben erwähnten Mißverhältnis der Resultate zu bilden, mit anderen Worten, ob dem Klima resp. der Außentemperaturein maßgebender Einflußauf die Menge der Hauttalgsekretion zuzusprechen sei.

Schon Krukenberg weist, allerdings ohne tatsächliche Unterlage, darauf hin, daß unbeständige Witterung eine Einwirkung auf die Produktion des Hauttalges haben könne und daß vielleicht zur Durchführung der Versuche wochenlang anhaltende, gleichmäßige Temperatur, wie sie in den Tropen herrsche, notwendig sei. Um diesem Umstande Rechnung zu tragen und die Frage experimentell zu studieren, unternahm ich an mir einen Versuch derart, daß ich, nachdem in einer Vorperiode bei einer Außentemperatur von weniger als $0^{\circ}$ die Wollwäsche 2 Tage lang getragen worden war, mich in einem Zimmer, welches auf $20^{\circ} \mathrm{R}$ erwärmt wurde, 2 Tage lang im Bett hielt. Auf analoge Art wurde an einer anderen Person ein zweiter Versuch durchgeführt. Der Erfolg war der, daß in dem einen Falle $3.46 g=1.73 g$ Hauttalg pro die, in dem anderen $2.57 \mathrm{~g}$ 
$=1.29 g$ pro die ausgeschieden wurde. In beiden Fällen betrug die täglicheHauttalgmengedemnach mehr, als die in der Vorperiode bestimmte Talgansscheidung bei der herrschenden Außentemperatur:

\begin{tabular}{|c|c|c|}
\hline \multicolumn{2}{|r|}{ Vorperiode } & Zimmerversuch bei $20^{\circ} \mathrm{R}$ \\
\hline $\mathbf{K}$. & $1.15 g$ pro die. & $1.73 g$ pro die \\
\hline G. & $1.05 g$ pro die & 1.29g pro die \\
\hline
\end{tabular}

Wir sehen also, daß bei Vornahme derartiger Versuche die jeweilige Höhe der Außentemperatur sebr wohl in Erwägung zu ziehen ist, und haben somit allen Grund anzunehmen, daß die uns anfänglich überraschenden Resultate, wenigstens zum Teil, in diesem bisher nicht beachteten Moment ihre Ursache gefunden haben dürften.

Später hat sich dann noch Birk mit Untersuchungen beschäftigt, in welchen er den Einfluß der Ernährung auf die Sekretion des Hauttalges studieren wollte, und ist dabei zu ähnlichen Resultaten wie den unserigen gekommen. Einer seiner 5 Versuche an Kindern fällt allerdings mit seiner enorm großen täglichen Hauttalgmenge von $4 \cdot 1-4 \cdot 5, g$ derart aus dem Rahmen des bisher Gewohnten und auch seiner übrigen 4 Versuche, daß hierfür wohl ein technischer Fehler verantwortlich zu machen sein dürfte. Jedenfalls erscheint uns dies plausibler, als die Annahme einer Seborrhoea oleosa, welcher Birk die Schuld an diesem Fehlresultat beimiBt. Betrachten wir die übrigen 4 Versuche, so ergeben sich bei ihnen Ausscheidungszahlen von 0.5 bis 1 bis $2.3 \mathrm{~g}$, gemessen an Kindern von 6 bis 10 bis 12 Jahren. Der Grund; weshalb manche Resultate den Wert von $1 g$ pro die nicht erreichen, ist mit einer gewissen Wahrscheinlichkeit darin $z u$ finden, daß die Versuche ja an Kindern angestellt worden sind, welche bekanntlich vor der Pubertät viel weniger Hauttalg produzieren als Erwachsene und bei denen erst nach Eintritt der Pubertät die Hautfettmengen steigen (Arnozan, Leubuscher). Wenn auch teilweise ein Einfluß der Ernährung auf die Hauttalgmenge im Rosenfeldschen Sinne konstatiert werden konnte, so traf dies jedoch nicht überall zu, und Birk kommt daher zu dem 
Schluß, daß eine gesetzmäßige Beeinflussung der Hauttalgausscheidung bei Kindern durch gewisse Nährstoffe nicht besteht.

Überblicken wir nunmehr das Gesamtresultat der bisherigen Versuche von Rosenfeld, Birk und mir, so lassen sich resümierend als Hauptergebnisse zwei feststellen:

1. Die tägliche physiologische Hauttalgausscheidung bält sich ungefähr innerhalb der Grenzen von $1-2 g$; sie ist ziemlich konstant, in der kalten Jahreszeit und bei Kindern etwas geringer als gewöhnlich.

2. Die Unterschiede in den Hauttalgzahlenbei kohlenhydratreicher Nabrung einerseits und fettreicher Nahrung andererseits sind, insbesondere in den Versuchender letztenZeit, nicht mehr in der früher angegebenen Prägnanz aufgetreten. Worauf diese Annäherung der Werte zurückzuführen ist, läßt sich für alle Experimente nicht mit Sicherheit sagen. Daß ein großer Teil wahrscheinlich durch technische Mängel sowie durch Nichtberücksichtigung der Außentemperatur erklärtwerden könnte, ist im vorhergehenden zum Teil experimentell zu beweisen versucht worden.

Besondere Beachtung verdient wohl die Feststellung Rosenfelds an zwei Diabetikern, nach welcher bei beiden übereinstimmend eine geringere, bis knapp an die untere Grenze der Norm und darunter reichende Ausscheidung des Hauttalges konstatiert wurde. In dem einen Falle, bei welchem sich eine ganz außergewöhnliche niedrige Hautfettzahl fand, war der Diabetes mit Furunkulose vergesellschaftet. Und wenn auch die Erklärung, welche nach dem Stande der damaligen Versuche dahin lautete, dab diese niedrigen Zahlen durch fettreiche Nahrung bei Diabetikern mit geringer Kohlenhydrattoleranz zustande gekommen wären, nach den eben skizzierten Darlegungen noch erst über einige Einwände hinaus zu sichern wäre, so bleibt doch die Tatsache eines Absinkens der Hautfettwerte bestehen. Durch die Aufdeckung dieser Beziehungen 
wird der Zusammenhang mit dem Ausgangspunkte dieser Abhandlung von den Diathesen und der funktionellen Prüfung wieder hergestellt, insofern nämlich, als wir bei einer exquisit diathetischen Erkrankung wie dem Diabetes eine Dermatose gefunden haben, welcher allem Anscheine nach eine Störung einer Hautfunktion zu grunde liegt, einer Funktion, deren physiologische Größe wir inzwischen durch das Experiment annähernd kennen gelernt haben. Von hier bedeutet es nur einen Schritt weiter zu der vom dermatologisch klinischen Standpunkte aus sehr wichtigen Frage, wie sich die Hauttalgproduktion bei anderen Dermatosen verhalten würde, bei welchen, wie bei der Akne vulgaris, eine Störung der Talgdrüsenfunktion auf diathetischer Grundlage schon seit jeher vermutet wurde. Da es leider nicht gelingt, experimentell Akne vulgaris zu erzeugen, bleibt, um dieser Frage nachzugehen, nichts anderes iibrig, als sich mit der dem Experiment zugänglichen medikamentösen (Brom-, Jod- etc.) Akne zu begnügen. In der Tat fand sich denn auch in einem schon von Rosenfeld angeführten Versuche eine Andeutung von Verminderung der Hauttalgsekretion. Die Zeit war aber zu kurz (3 Tage), es trat keine Akne auf und der Unterschied gegenüber der Norm war so gering $(2 \cdot 2 \mathrm{~g}$ gegen $2.05 \mathrm{~g}$ ), daß zur Sicherung des Ergebnisses unbedingt ein länger dauerndes Experiment erforderlich war. Und so unternahm ich an mir die folgenden Versuche:

Nachdem in einer viertägigen Vorperiode bei freier Kost der täglich abgeschiedene Hauttalg festgestellt war, wurde der eigentliche Versuch angefangen, in dem ich, ebenfalls bei freier Kost, außerdem noch täglich 4-5 g Bromnatrium in etwas Wasser gelöst zu mir nahm. Nach 4-5 Tagen zirka trat starke Akne mit Pustelbildung im Gesicht und auf dem behaarten Kopfe auf und verbreitete sich im Verlauf der nächsten Tage über den übrigen Körper. Nach 10 Tagen wurde dieser Versuch abgebrochen und eine dritte Untersuchungsperiode von 3 Tagen hinzugefügt, innerhalb welcher aber kein Brom mehr genommen wurde. In dieser Zeit bestand die Akne fort. Das Ergebnis war evident. Während ich in der Vorperiode von 4 Tagen $4.94=1.23 \mathrm{~g}$ Fett pro die ansschied, wurden in der 10 tägigen Bromperiode im 
ganzen nur $7 \cdot 3 g=0.7 \mathrm{~g}$ Hauttalg pro die produziert, also etwas mehr als die Hälftedertäglichen Hauttalgmenge zur Zeit der Vorperiode. In der nun folgenden 3tägigen Versuchszeit zeigte es sich, daß die Hautfettzahlen zwar in die Höhe gegangen waren, aber immer noch nicht die untere Grenze der Norm erreicht hatten. Es wurden im ganzen $2.83 g=0.94 g$ Hautfett pro die gefunden. Die tabellarische Übersicht der Zahlen des Bromversuches stellt die Ergebnisse folgendermaßen dar:

\begin{tabular}{|c|}
\hline Vorperiode . . . . . 1.23 g pro die \\
\hline $\begin{array}{l}\text { Bromversuch. } \cdots \cdots \cdot 0.73 g \text { pro die } \\
\quad(4-5 g \text { Br. täglich) }\end{array}$ \\
\hline $\begin{array}{l}\text { Nachperiode . . . . } 0.94 g \text { pro die } \\
\text { (kein Br.) }\end{array}$ \\
\hline
\end{tabular}

Man kann das Resultat dieses Versuches somit dahin aussprechen, dab während der Zeit, in welcher Akne durch Brom erzeugt wurde, die Produktion des Hautfettes erheblich vermindert war.

Dieses überraschende Resultat ist vielleicht geeignet, die bisher widerspruchsvollen Anschauungen über die Entstehung der Bromakne in gewisser Beziehung zu klären. Der eine Teil der Autoren führt die Bromakne auf eine rein chemische Ursache zurück, wonach das in den Talgdrüsen ausgeschiedene Brom der Faktor sein soll, welcher allein den entzündlichen krankhaften Prozeß erzeugt. Dieser Annahme stehen entgegen die Ergebnisse vieler Untersucher, welche gar kein oder nur sehr wenig Brom im Inhalt des Akneeiters gefunden haben. Auch wir haben das während der Bromzeit gewonnene Hautfett mit dem Jolles schen Reagens (Dimethylphenylendiaminchlorhydrat) auf das Vorhandensein von Brom geprüft, vermochten aber nur eine ganz schwach positive Reaktion nachzuweisen. In jedem Falle sind die ausgeschiedenen Brommengen zu verschwindend gering, um eine derartige Affektion wahrscheinlich zu machen, so daB wohl von der Annahme einer rein chemischen Entzündung Abstand zu nehmen ist. Anders dagegen verhält es sich mit der Anschauung der meisten anderen Dermatologen, wie Neisser, Jarisch, Ehrmann (in 
Exper. und klin. Beiträge zur Frage der Hauttalgsekretion. 703

Mraceks Handbuch) etc., welche den Schwerpunkt bei der Entstehung der Bromakne darauf legen, daß es die überall in den Follikeltrichtern sitzenden Bakterien sind, welche den entzündlichen Prozeß erzeugen, und daß diese Entzündung erst durch das in den Talgdrüsen ausgeschiedene Brom, sei es durch Reizung oder Schädigung des Gewebes, begünstigt wird. Aber hier wie dort wird immer mit der Sekretion von Brom in die Talgdrüsen gerechnet, was jedoch notgedrungen zu einem Widerspruche führen muß, da es nach den eben erwähnten Darlegungen ganz unwahrscheinlich ist, daß Brom in irgendwie nennenswerten Mengen in den Talgdriisen zur Ausscheidung gelangt. Die so entstehende Lücke läßt sich durch Heranziehung des aus unseren Versuchen gewonnenen Resultates ungezwungen ausfillen. Auch wir schuldigen die ubiquitären, in den Follikeln befindlichen Hautbakterien an, die Entzündung zu verursachen, emanzipieren uns aber von der Annahme der Sekretion von Brom in die Talgdrüsen und glauben vielmehr, da $B$ es die durch das Brom bedingteVerringerung des Hauttalges ist, welche die Entzündung begünstigt.

Wie der Mechanismus dieser Wirkung zustande kommt, das zu erklären ist auf verschiedene Weise möglich. So sucht Rosenfeld diesen Zusammenhang dadurch verständlich zu machen, dafi er annimmt, der im Follikeltrichter sich befindende Hauttalg brauche infolge der stockenden Hauttalgsekretion längere Zeit, um auf die Oberfläche der Haut zu gelangen, als unter normalen Verhältnissen, und dadurch sei den Hautbakterien bessere Gelegenheit gegeben, sich am oberen Ende des Drüsenausführungsganges einzunisten und eine Entzündung hervorzurufen.

Ein weiterer Erklärungsversuch wäre der, daß der Hauttalg, infolge der Körpertemperatur in etwas flüssigem Zustande, jedes einzelne Bakterium einhüllt und, $d a$ er einen für Bakterien sehr ungünstigen Nährboden abgibt (Linser) - wenn er nicht gar selbst bakterizid ist -, durch diese so gebildete entwicklungshemmende Isolierschicht den Erreger von dem ihm günstigen Nährsubstrat fernhält. Geschieht dies unter normalen Sekretionsverhältnissen in völlig ausreichendem Maße, 
so wird eben bei verringerter Talgausscheidung eine genügende Schutzwirkung nicht mehr zustande kommen.

Schließlich wäre auch noch daran zu denken, daß möglicherweise dem Hauttalg direkt, der ja infolge seiner chemischen Konstitution dem Lanolin außerordentlich nahe steht, ebenfalls bakterizide Eigenschaften zukoumen, wie sie Gottstein vom Lanolin wahrscheinlich gemacht hat, und daß es somit nach Einschränkung der Bildung bakterizider Substanz naturgemäß viel leichter zur Infektion des Follikels kommen kann.

Es will uns scheinen, daß die Furunkulose der Diabetiker mit diesen Verhältnissen manche Analogien aufweist, und die Vermutung, daß dem Hautfett in den Mengen, in denen es normalerweise produziert wird, als Schutzstoff gegen bakterielle Infektionen eine wesentliche Bedeutung beizumessen ist, gewinnt an Wahrscheinlichkeit. Fällt durch den Einfluß irgendwelcher Faktoren ein Teil dieser normalen Fettmenge fort, wie sich das für die Bromakne und beim Diabetiker hat nachweisen lassen, so kann es unter Mitwirkung ubiquitärer Bakterien in dem einen Falle zu Akne, in dem anderen zu Furunkulose kommen.

Hieran dürfte sich eine Gruppe von Dermatosen auf „ar-

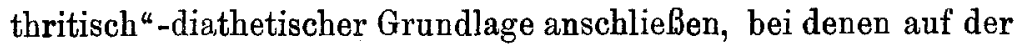
Basis einer latenten pathologischen Disposition und im Verein mit einer Gelegenheitsursache - wie Bl o c h es definiert - eine krankhafte Reaktion der Haut ausgelöst wird. Ich möchte dazu eine gewisse Form von Ekzemen bei Fettleibigen rechnen, bei denen die pathologische Disposition in der konstitutionellen Anomalie gegeben ist und bei welchen die Gelegenheitsursache die Staphylokokken bilden, welche ja nach den Untersuchungen von Bruck und Hidaka bei allen Ekzemen eine wesentliche Rolle zu spielen imstande sind. Diese trotz energischer Behandlung rezidivierenden Ekzeme sehen wir nach Entfettung, besonders durch kohlenhydratreiche, fettarme Kost rasch abheilen und längere Zeit rezidivfrei bleiben. Möglicherweise gehören auch in diese Gruppe die Ekzeme der pastösen, exsudativ-diathetischen Säuglinge hinein, deren fortschreitende Heilung durch Abmagerung bewirkende Maßnahmen man ebenfalls konform mit der Gewichtsverminderung des Säuglings beobachten kann. 
Exper. und klin. Beiträge zur Frage der Hauttalgsekretion. 705

Umgekehrt liefert zu dieser Frage die sehr interessante klinische Beobachtung von A. Czerny an älteren Kindern einen wichtigen Beitrag, welche durch die Exaktheit, mit welcher sie jedesmal eintrat, fast die beweisende Kraft eines Laboratoriumexperimentes besitzt. Czerny erhöhte auf den Rat eines Kollegen die auf der Tuberkulosestation seiner Klinik übliche Lebertrandosis und gab den Kindern so viel, als sie ohne nachweisbare Störungen vertrugen. Dies hatte zur Folge, daB ein Kind nach dem anderen Gesichts-und Koptekzem bekam, eine Erscheinung, welche er auf seiner Tuberkulosestation niemals vorher beobachtet hatte. Nach Sistierung der Lebertranverabreichung verschwanden die Ekzeme wieder.

Czerny spricht sich in folgender Weise aus - und ich zitiere seine Sätze wörtlich als beste Illustration für unsere Anschauungen :

„Daß das Milchfett, wenn es im Übermaßgegeben wird, einen ungünstigen EinfluB auf die Symptome der exsudativen Diathese bei Säug. lingen ausübt, ist genügend bekannt. Unsere angeführte Erfahrung beweist aber, daß manauch bei älterenKindern durchextremeFetternährung Symptome dieser Diathese provozieren kann."

$\mathrm{Ob}$ aber die Beziehungen zwischen Hauttalgsekretion und Bakterieninvasion, wie sie sich bei der Bromakne vorgefunden haben, nun auch auf die Akne vulgaris zu übertragen und damit zu einer Erklärung derselben heranzuziehen sind, erscheint mir noch immer fraglich. Denn abgesehen daron, daß diese beiden Affektionen der Talgdrüsen klinisch als ganz verschiedene Erkrankungen anzusprechen sind, könnte hier sogleich der Einwand erhoben werden, daß meistens schon der bloße Aspekt der Aknekranken lehre, $d a b$ sie nicht an einem $z u$ wenig, sondern an einem zu viel an Hauttalg litten. Denn oft erscheint das Gesicht dieser Leute geradezu wie in Fett getaucht, und man hat daher diesen Zustand mit Seborrhoea oleosa gekennzeichnet, um hierdurch die Überproduktion an Hautfett auszudrücken. Einen strikten wissenschaftlichen Beweis für diese Annahme zu 
liefern, dürfte schon deshalb nicht leicht sein, weil wir bis beute noch über kein Mittel verfügen, durch welches wir imstande sind, wie bei den Schweißdrüsen, direkt mit Energie auch auf die Talgdrüsen einzuwirken, sei es im Sinne einer Anregung oder Ausschaltung ihrer Tätigkeit. Aber vielleicht handelt es sich bei der Seborrhoea oleosa gar nicht um eine echte Vermehrung der Hauttalgsekretion, sondern vielmehr um eine Verdünnung normaler oder sogar unternormaler Hautfettmengen durch Wasseraufnahme aus dem fettarmen oder fettlosen Schweiß. Daß diese Möglichkeit besteht, geht aus den Berichten Linsers herror, welcher vor allem die große Wasseraufnahmefähigkeit der (Hautfett-) Extrakte betont, die ähnlich wie das Lanolin imstande seien, etwa die gleiche Gewichtsmenge aufzunehmen.

Dem gegenüber gibt uns eine andere Untersuchung Linsers, welcher bei Seborrhoea oleosa die tägliche Fettabscheidung der Gesichtshaut gemessen hat, einen einigermaßen befriedigenden Anhaltspunkt. $\mathrm{Er}$ ließ das Gesicht täglich morgens mit einem in Petroläther getauchten Wattebausch abreiben, das Material 4 Wochen sammeln und dann extrahieren. $\mathrm{Da}$ der Ätherextrakt zirka $2 g$ wog, würde in diesem Falle die tägliche Ausscheidungsmenge $0.07 \mathrm{~g}$ betragen. Es erhebt sich nun die Frage: Überschreitet diese von Linser gefundene Zahl das normale Maß der vom Gesicht täglich ausgeschiedenen Hautfettmenge? Zur Beantwortung dieser Frage war also nötig, vorher die physiologische Hauttalgzahl zu kennen, wobei gleichzeitig von Interesse war, festzustellen, ob das Gesicht, welches ja außerordentlich reich an wohlausgebildeten Talgdrüsen ist, im Verhältnis zum übrigen Körper schon normalerweise mehr Fett sezerniert, eine Behauptung, welche z. B. Arnozan seinerzeit aufgestellt hat.

$\mathrm{Zu}$ diesem Zwecke ließ ich mir täglich zweimal das Gesicht mit Wattebäuschen, welche in Äther getaucht waren, abreiben. Die Bäusche wurden gesammelt, getrocknet und in der üblichen Weise extrahiert. Die $\mathrm{Zahl}$, welche ich mit dieser Methode innerhalb von 7 Tagen $^{1}$ ) erhielt, betrug $0.32 \mathrm{~g}=$

1) Selbstverständlich wurde auf jede sonstige Reinigung des Gesichtes, auch auf Rasieren verzichtet. 
$0.046 \mathrm{~g}$ p ro die. Auf Grund dieser Werte lälst sich ein annäherndes Urteil über die Talgsekretion der Gesichtshaut im Verhältnis zu derdes Gesamtkörpers gewinnen. Nach $\mathrm{Me}$ eh und Weidenfeld verhält sich die Oberfläche des Kopfes zu der des übrigen Körpers wie 1:21, demnach die Oberfläche des Gesichtes, für welche man wohl die Hälfte der Kopfoberfläche in Anspruch nehmen darf, wie 1:42. Setzt man nun den oben gefundenen Wert in diese Gleichung ein, so erhält man die $\mathrm{Zahl} 1.96$ pro die, eine Zahl, welche wir schon früher als normalen Verhält$n$ issen entsprechend kennen gelernt haben. Hiernach hat es den Anschein, wie wenn die Gesichtshaut nicht mehr, sondern nur ebensoviel Hauttalg produzierteals die des übrigen Körpers.

Setzen wir hingegen die von Linser gefundene $\mathrm{Z}$ ahl von $0.07 g$ in unsere Gleichung ein, so sehen wir, daß der sich daraus ergebende Wert ron $2.9 \mathrm{~g}$ prodie das physio$\log$ is che MaB überschreitet. Wir würden also auf Grund der mit unserer Methode gewonnenen Zahlen, die ein annäherndes Urteil zulassen, annehmen dürfen, $d a b$ sich bei $d$ er mit Seborrhoea oleosa kombinierten Form der Akne vulgaris der klinische Befund mit dem chemischen deckt, mit anderen Worten, daB die Seborrhoea oleosa möglicherweise mit einer Vermehrung der Hauttalgsekretion einhergeht.

Nichtsdestoweniger fügt sich diese Erscheinung zwanglos in den Rahmen unserer Vorstellungen von den Beziehungen zwischen Hauttalgsekretion und Bakterieninvasion. Man könnte sich ganz gut denken, daß bei der Akne vulgaris auch die normaler weise sezernierte Hautfettmenge der Epidermis eben keinen ausreichenden Schutz mehr bietet, so daß sich der Organismus durch eine Überproduktion an Hautfett helfen muß. Wir würden demnach hierbei eine Art natürlicher Abwehrvorrichtung vor uns haben, in dem Sinne, daß der reichlich fließende Hauttalg teils mechanisch die Bakterien entfernen, teils den Nährboden in einer für die Krankheitserreger ungünstigen Weise beeinflussen soll, indem er die Haut in dünner Schichte isolierend überzieht. Daß dieser Heilversuch nur schwer zum 
Ziele führen kann, daß er vielmehr nur rein prophylaktische Bedeutung besitzen dürfte, liegt auf der Hand. Man wird einer vermehrten Hauttalgsekretion natürlich ebenso wenig eine akut einsetzende, antibakterielle, entziundungsverhindernde Wirkung beimessen können, wie man erwarten wird, daß beispielsweise ein Furunkel oder Karbunkel eines Diabetikers durch diätetische Maßnahmen allein zur Abheilung gelangt. $\mathrm{DaB}$ aber der Hauttalgsekretion eine langsam wirkende antiparasitäre Kraft zuzusprechen ist, lebren mancherlei klinische Beobachtungen. So sehen wir jene bekannte Form der Furunkulosis, welche ebenso wie die Akne in der Pubertätszeit, einer Zeit mit geringer Hauttalgsekretion, auftritt, in späteren Jahren ausheilen, wenn die Fettproduktion ihr normales Maß erreicht hat. Des weiteren möchte ich hier die Beobachtung Blochs im Sinne unserer Thesen anführen, daß "gewisse Dermatomykosen (Farus, Mikrosporie, Trichophytie), die fast nur bei Kindern auftreten, und während sie sonst therapeutisch kaum zu beeinflussen sind, mit Beginn der Pubertät spontan auszuheilen pflegen".

Es ist mir sehr wahrscheinlich, daß auch in diesen Fällen die Vermehrung des Hauttalges in der Pubertät bei dieser späten spontanen Ausheilung eine entscheidende Rolle spielt. Alle diese Erörterungen würden mit der Annahme stimmen, dab der Hauttalg in vermehrter Menge, wie wir ihn bei der Seborrhoea oleosa vorfinden, nicht, wie man bisher annahm. die Ursacheder begleitenden Akne darstellt, sonderndaB wir im Gegenteil in ihm aller Wabrscheinlichkeit nach eine zwar schwache, aber immerhin deutlich erkennbare AbwehrmaBregel des Organismus zu sehen haben.

\section{Literatur.}

1. Birk. Monatsschr. für Kinderheilkunde.'1909. Bd. VIII.

2. Bloch. Ref. für den 28. Kongr. für inn. Med. Wiesbaden 1911.

3. Bruck. Arch. f. D. u. Syph. 1909 u. Berl. klin. Woch. 1910.

4. Brack und Hidaka. Arch. für Derm. n. Syph. 1910. Bd. C.

5. Czerny. Therapie der Gegenwart, Februar 1912.

6. Krukenberg. Chemische Untersuchungen zur wissenschaftlichen Medizin. 1886.

7. L e ubuscher. Verhandl. d. Kongr. tür innere Medizin. 1899.

8. Linser. Über den Hauttalg beim Gesunden und bei einigen Hauterkrankungen. Habilitationsschrift, Tübingen 1904.

9. Rosenfeld. Zentralbl. für innere Medizin. 1906.

10. Wechselmann und Loewy. Berl. klin. Woch. 1911. Nr. 30. 